International Journal of Pure and Applied Mathematics

Volume 89 No. 3 2013, 433-438

ISSN: 1311-8080 (printed version); ISSN: 1314-3395 (on-line version)

url: http://www.ijpam.eu

doi: http://dx.doi.org/10.12732/ijpam.v89i3.13

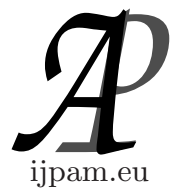

\title{
STUDY BASICITY OF ROOT FUNCTIONS OF THE SCHRODINGER OPERATOR WITH
}

\section{A NON-LOCAL PERTURBATION}

N.S. Imanbayev ${ }^{1 \S}$, B. Kalimbetov ${ }^{2}$, A.M. Sarsenbi ${ }^{3}$

${ }^{1,2}$ Department of Mathematics

Akhmet Yasawi International Kazakh-Turkish University

29, Sattarkhanov Str., 161200, Turkistan, KAZAKHSTAN

${ }^{3}$ Department of Mathematics

M.O. Auezov South Kazakhstan State University

5, Tauke Khan Str., 160012, Shymkent, KAZAKHSTAN

\begin{abstract}
The asymptotics of the eigenvalues is received, and conclusions about the stability and instability of the basis property of the system of eigenfunctions and associated functions of the Schrodinger operator for various occasions of the disarmed regularity of the boundary conditions are done. In the paper there is considered the Samarsky - Ionkin spectral problem for the Schrodinger equation with an integral perturbation in the boundary conditions. It is assumed that the unperturbed problem has a system of eigenfunctions forming a Riesz basis in $L_{2}(0,1)$. It is shown that the basis property of the systems of root functions of a problem can be varied under any arbitrarily small variation of the kernel of the integral perturbation.
\end{abstract}

AMS Subject Classification: 80M22, 34B10

Key Words: basis, Samarsky-Ionkin problem, perturbation, eigenfunctions, eigenvalues

Received: October 10, 2013

(c) 2013 Academic Publications, Ltd. url: www.acadpubl.eu

${ }^{\S}$ Correspondence author 
Consider operator $L_{1}$, given by the differential expression

$$
L_{1} u=-u^{\prime \prime}+q(x) u=\lambda u, \quad x \in(0,1), \quad q(x) \in C[0,1],
$$

and regular, but not strongly regular boundary conditions [1] with an integral perturbation

$$
\begin{aligned}
& U_{1}(u) \equiv a_{11} u^{\prime}(0)+a_{12} u^{\prime}(1)+a_{13} u(0)+a_{14} u(1)=0, \\
& U_{2}(u) \equiv a_{23} u(0)+a_{24} u(1)=\int_{0}^{1} \overline{p(x)} u(x) d x, p(x) \in L_{2}(0,1) .
\end{aligned}
$$

Here $U_{j}(u)$ - are independent linear forms with complex-valued constant coefficients, satisfied conditions of not strong regularity

$$
\left|a_{11}\right|+\left|a_{12}\right| \neq 0, a_{11} a_{24}+a_{12} a_{23}= \pm\left[a_{11} a_{23}+a_{12} a_{24}\right] \neq 0 .
$$

In [2] they proved that the system of eigenfunctions and associated functions (E and AF) of the problem (1) - (2) forms a Riesz basis [3] with the brackets in any integral perturbation of boundary conditions, and in strong regularity of the boundary conditions $\mathrm{E}$ and AF form a Riesz basis. In particular case of integral perturbation of periodical boundary conditions for multiple differentiation operator instability of the basis property of $\mathrm{E}$ and AF is investigated in $[4]$.

Note, that in [5] questions about stability of basic properties of the periodic problem for the loaded equation with the load of the form $p(x) \nu(0)$ are studied.

We get the following theorem.

Theorem 1. Let unperturbed operator $L_{0}$ (when $p(x) \equiv 0$ ) have eigenvalues $\lambda_{k}^{0}$ and $E$ and $A F$ forming a Riesz basis in $L_{2}(0,1)$.

Then the characteristic determinant of the operator $L_{1}$ with the perturbed regular boundary conditions (2) is represented as:

$$
\begin{array}{r}
\Delta_{1}(\lambda)=\Delta_{0}(\lambda)\left[1-\sum_{k=1}^{\infty} \overline{a_{k 0}}\left(\frac{V_{3}\left(\nu_{k 0}\right)}{\lambda-\lambda_{k}^{0}}-\frac{\sqrt{\lambda_{k}^{0}} \overline{V_{3}\left(\nu_{k 1}\right)}}{\left(\lambda-\lambda_{k}^{0}\right)^{2}}\right)\right. \\
\left.-\sum_{k=1}^{\infty} \overline{a_{k 1}} \frac{V_{3}\left(\nu_{k 1}^{0}\right)}{\lambda-\lambda_{k}^{0}}\right],
\end{array}
$$


where $\Delta_{0}(\lambda)$ - is a characteristic determinant of the operator $L_{0} ; V_{3}(\nu)$ - are linear homogeneous forms emerging during the construction of the boundary conditions of dual unperturbed problem $L_{0}^{*} ;\left\{\nu_{k 1}^{0}, \nu_{k 0}^{0}\right\}-\mathrm{E}$ and AF of the dual unperturbed problem, $a_{k 1}, a_{k 0}$ - Fourier coefficients of the biorthogonal expansion of the function $\overline{p(x)}$ by the following system:

$$
\overline{p(x)}=\sum_{k=1}^{\infty}\left[\overline{a_{k 1}} \nu_{k 1}^{0}+\overline{a_{k 0}} \nu_{k 0}^{0}\right] .
$$

Due to this representation of the characteristic determinant of the problems (1) - (2), we obtain the asymptotic behavior of the eigenvalues, and we can draw conclusions about the stability and instability of basis property of the E and AF system for various occasions of the disarmed regularity of the boundary conditions.

As an example take the perturbed Samarskii - Ionkin problem for multiple differentiation operator, i.. when $q(x) \equiv 0$ :

$$
\begin{gathered}
u^{\prime}(0)=u^{\prime}(1), u(0)=\int_{0}^{1} \overline{p(x)} u(x) d x, \\
u^{\prime}(0)=u^{\prime}(1)+\int_{0}^{1} \overline{p(x)} u(x) d x, u(0)=0,
\end{gathered}
$$

where $p(x) \in L_{2}(0,1)$.

One of the features of these problems is the fact that the dual problems to (1) - (2a) and (1) - (2b) are the spectral problems for the loaded differential equations:

$$
\begin{aligned}
& \left\{\begin{array}{l}
l^{*}(\nu)=-\nu^{\prime \prime}(x)+p(x) \nu^{\prime}(0)=\bar{\lambda} \nu(x), \\
V_{1}(\nu) \equiv \nu^{\prime}(1)=0, \\
V_{2}(\nu) \equiv \nu(0)-\nu(1)=0,
\end{array}\right. \\
& \left\{\begin{array}{l}
l^{*}(\nu)=-\nu^{\prime \prime}(x)+p(x) \nu(0)=\bar{\lambda} \nu(x), \\
V_{1}(\nu) \equiv \nu^{\prime}(1)=0, \\
V_{2}(\nu) \equiv \nu(0)-\nu(1)=0 .
\end{array}\right.
\end{aligned}
$$

Characteristic determinant of the problem (1)-(2b):

$$
\Delta_{1}(\lambda)=\Delta_{0}(\lambda) \cdot\left[1+4 \sqrt{2} \pi \sum_{k=1}^{\infty} \overline{a_{k 0}} \cdot \frac{k}{\lambda-(2 k \pi)^{2}}\right],
$$


Characteristic determinant of the problem (1)-(2b):

$$
\begin{aligned}
\Delta_{1}(\lambda)=\Delta_{0}(\lambda) & {[1} \\
& \left.-4 \sqrt{2} \cdot \sum_{k=1}^{\infty}\left(\pi \overline{a_{k 0}} \frac{k}{\left[\lambda-(2 k \pi)^{2}\right]^{2}}+\overline{a_{k 1}} \frac{k}{\lambda-(2 k \pi)^{2}}\right)\right],
\end{aligned}
$$

where

$$
\begin{gathered}
\Delta_{0}(\lambda)=1-\cos \sqrt{\lambda} \\
\overline{p(x)}=\sum_{k=1}^{\infty} \overline{a_{k 0}} \cdot 2 \sqrt{2} \cdot(1-x) \cdot \sin 2 k \pi x+\sum_{k=1}^{\infty} \overline{a_{k 1}} \cdot 4 \sqrt{2} \cos 2 k \pi x .
\end{gathered}
$$

The function $\Delta_{1}(\lambda)$, given by the formula (3) and (3b), is a whole analytic function of a variable $\lambda$.

By the formula (3) we have two rows of the eigenvalues of the perturbed problem (1)-(2):

$$
\begin{gathered}
\lambda_{1}^{(1)}=\lambda_{k}^{0}=(2 k \pi)^{2} \\
\lambda_{k}^{(2)}=\left[2 k \pi+\overline{a_{k 0}}\left(\sqrt{2}+\underline{\underline{O}}\left(\frac{1}{\sqrt{k}}\right)\right)\right]^{2} .
\end{gathered}
$$

Note that E and AF systems of the perturbed problems (1)-(2), (1)-(2b) and $\mathrm{E}$ and AF systems of the unperturbed Samarskii - Ionkin problem (forming a Riesz basis) are differ from each other only a finite number of the first members. Consequently, E and AF systems of the perturbed problems (1)-(2), (1)-(2b) also form Riesz basis in $L_{2}(0,1)$.

Set of the functions $p(x)$, represented as a kind of $(4 \mathrm{~b})$, is dense in $L_{2}(0,1)$.

Theorem 2. Set of the functions $p(x) \in L_{2}(0,1)$, such that $E$ and $A F$ systems of the perturbed problems (1)-(2) and (1)-(2b) form a Riesz basis in $L_{2}(0,1)$, is dense in $L_{2}(0,1)$.

To show that the basis property of the $\mathrm{E}$ and $\mathrm{AF}$ system of the perturbed problems (1) - (2a), (1) - (2b) is unstable for an arbitrarily small integral perturbation of the boundary condition (2a), we find the eigenfunctions of (1) $(2 \mathrm{a})$, and for the boundary condition (2b), we find the eigenfunction of the dual problem (2b), corresponding to the eigenvalues $\lambda_{j}^{0}=(2 j \pi)^{2}$, i.e.

$$
u_{j}^{1}(x)=C_{1}\{\cos (2 j \pi x)
$$




$$
\begin{array}{r}
+\frac{\sqrt{2}}{a_{j 0}}\left[1-\frac{\sqrt{2}}{4 \pi} \cdot \frac{\overline{a_{j 0}}}{j}+\frac{\sqrt{2}}{\pi} \sum_{\substack{k=1 \\
k \neq j}}^{\infty} \overline{a_{k 0}} \frac{k}{j^{2}-k^{2}}\right] \sin (2 j \pi x), \\
\nu_{j 0}(x)=C_{1}\left\{\cos (2 j \pi x)-\left[2 \sqrt{2} \cdot \overline{a_{j 1}}+\frac{O}{=}\left(\frac{1}{j^{2}}\right)\right] \sin (2 j \pi x)\right. \\
+\int_{0}^{\chi} p(\xi) \frac{\sin (2 j \pi(x-\xi)) d \xi}{2 j \pi} .
\end{array}
$$

We choose the constant $C_{1}$ by the biorthogonal condition for $(5),\left(u_{j}^{1}(x), \nu_{j}^{1}(x)\right)=$ 1 , for $\left(5 \mathrm{~b}^{*}\right),\left(u_{j 0}(x), \nu_{j 0}(x)\right)=1$.

Then, due to the Young Theorem [6], for the problems (1)-(2), $\lim _{j \rightarrow \infty}\left\|u_{j}^{1}\right\|$. $\left\|\nu_{j}^{1}\right\|=\infty$, and for the problems (1)-(2b), $\lim _{j \rightarrow \infty}\left\|u_{j 0}\right\| \cdot\left\|\nu_{j 0}\right\|=\infty$.

It means condition of uniform minimal [7] of the $\mathrm{E}$ and AF system does not hold, and therefore it does not form a regular basis in $L_{2}(0,1)$.

This fact we can establish in the following

Theorem 3. Set of the functions $p(x) \in L_{2}(0,1)$, such that $E$ and $A F$ systems of the perturbed problems (1)-(2) and (1)-(2b) do not form a regular basis in $L_{2}(0,1)$, is dense in $L_{2}(0,1)$.

Remark. Dual operators simultaneously have the property of Riesz basicity of root functions.

The results of the paper, in contrast to [2], demonstrate the instability and stability of the basis property of root functions of the problem with an integral perturbation of the boundary conditions, which are regular, but not strongly regular. In conclusion, we thank the member of the National Academy of Sciences of Kazakhstan, Doctor of Physical and Mathematical Sciences, Professor M.A. Sadybekov for discussing the results of the paper.

\section{References}

[1] M.A. Naimark, Linear Differential Operators, Nauka, Moscow (1969), 352 pp. 
[2] A.A. Shkalikov, On the basis of eigenfunctions of ordinary differential operators with integral boundary conditions Vestnik MGU. Series of Math. and Mech., 6 (1982), 12-21.

[3] A.I. Sarsenbi, Theory about Basicity of Root Vectors of Non-Selfadjoint Linear Differential Operators in a Hilbert Space, Shymkent (2009), 267 pp.

[4] A.S. Makin, On nonlocal perturbation of the periodic problem for the eigenvalues, Differential Equations, 42, No. 4 (2006), 560-562.

[5] N.S. Imanbayev, I.A. Sadybekov, Basic properties of the root functions of loaded differential operators of the second order, Doklady NAN RK., 2 (2010), 11-13.

[6] G.G. Hardy, J.E. Littlewood, G. Polya, Inequalities, BK, Moscow (1948), 456 pp.

[7] S.G. Kreyn, Editor, Functional Analysis (1972). 